\title{
A Survey on Energy Efficiency and Cluster Handling in Real Time Wireless Sensor Networks
}

\author{
Janish $^{1}$ Dr. Mamta Katiyar ${ }^{2}$ \\ ${ }^{1,2}$ Department of Electronics and Communications, Maharishi Markandeshwar University, Mullana, Ambala
}

\begin{abstract}
Wireless networking technology in recent years has seen a thriving development. Wireless sensor networks (WSN) has remote environmental monitoring and target tracking as an important application. This makes sensors smaller and cheaper especially in recent years. These sensors form a network in the wireless interface with which they can communicate with each other. Design of WSN relies heavily on the application such as environment, and it also must consider design objectives, costs, hardware, and factors in which system is constrained. There are so many aspects involved in the wireless sensor networks which need to be readdressed or reviewed for further improvement, generally these are Clustering, Energy saving concepts and Network lifetime. Various researches and journals has been presented so far. In the current paper we are going to enlighten these aspects through survey of research studied that has being done.
\end{abstract}

Keywords: WSN, Clustering, Network Lifetime, Residual energy, power consumption

\section{Introduction}

Smart manufacturing environment, utilities, industrial, home, shipboard, and transportation systems automation represent the next evolutionary step in the development. Like any sentient organism, smart environment with the real world depends first and foremost on sensory data. Sensitive data in distributed locations comes from multiple sensors of different modalities. Smart environment as well as about its internal workings need information about surroundings. Challenges in the hierarchy of relevant trace amounts, monitoring and data collection, to assess and evaluate information, to prepare meaningful user displays, and decision-making and performance alarms are enormous. Information needed by smart environments are provided by distributed wireless sensor networks, are responsible for sensing as well as for the first stage of the processing hierarchy. Sensor networks shows the importance in the recent funding initiatives, DARPA sensit programs, military programs, and is highlighted by including NSF program announcements.

There are so many schemes that are involved which lets the system to work more efficient and fast, also uses less resources and power to send maximum data from one node to another node. So these types of systems organization needs extra handling and smart techniques which leads to give good results, Because using these technique may create so many pros and cons. Such as we can take the example of Static and Dynamic clustering. In case of Static clustering, there is less overhead but it lead to energy drain of nodes in the network where as in case of dynamic clustering energy can be preserved but it increases the overhead. So there are key points such as energy and cluster handling in real time scenario need to be readdressed and reviewed. Following are the key challenges which are involved:

\section{Challenges For Wireless Sensor Networks}

1. Power Consumption: In wireless sensor networks (WSNs), different applications require long life run while sensor nodes has limited energy storage capacity so for long lifetime and to reduce power consumption these factors has led to explore new horizons. The increase in lifetime of the sensor nodes, circuit and protocol must be energy efficient and less power consuming so that they asses and predict energy consumption and make priori reactions.

2. Cluster Management: Clustering technique is an important for reducing the energy consumption and to extend the lifetime of a sensor network it is used. It can also increase the scalability of network. Wireless sensor network nodes are homogeneous in all areas that researchers believe, but some nodes to prolong the lifetime of the WSN and its reliability can be of different energy. Sensor network can be made scalable, for this groups can be created by assembling nodes i.e each group is called cluster. Each cluster leader, often referred as clusterhead. $\mathrm{CH}$ elected by a sensor in a cluster or network designer can already assign it. Cluster membership can be fixed or variable. A number of special scalability and clustering algorithms for WSNs is designed for efficient communication.

3. Efficient Data Transfer: A wireless sensor networks (WSNs) in dense deployment of sensor nodes, sensor spatially correlated and they sense the same information and can send the same information to the sink node. If each sensor node transmit redundant data is the waste of energy. Moreover, sensor nodes need more energy than other nodes in handling the data near the sink node. Then, an energy-efficient data 
transmission system which reduces unnecessary data transmission and saves energy node to sink nodes always necessary for proper data transfer.

4. Network Lifetime: Network that constitutes the sensor nodes strongly depends on the lifetime of every single node. This fact is not depend on how lifetime of the network define. At the end of each definition can be reduced to the question of when the individual nodes fail. Thus, if the nodes are not accurately predicted lifetimes, it is possible to derive metric lifelong network deviates from an uncontrollable manner. So it should be obvious that an accurate and consistent modeling of the nodes is very important. Lifetime network is directly related to the battery life of node until one of the first node dies due to lack of energy.

5. Congestion Control: WSN can have periodic or infrequent traffic. Such network congestion does not seem to be a big problem. However, the crowd is more in demanding and larger systems that is a problem for audio, video processing and many base stations (making more cross traffic) and can be a major issue. Even in systems with a single base station, base station to base station traffic congestion is a serious problem since converges at the base station.

\section{Energy Efficient Cluster Handling In Real Time WSN}

In a Sensor network, clustering technology enables it to work more efficiently. This increases the sensor network lifetime and energy consumption in it. The main role of the cluster head node is to provide data communication between the base station and sensor nodes efficiently. So energy in the cluster head should be higher than the energy in other nodes as it also aggregates the data.

Cluster-based routing protocols makes the operation of wireless sensor network highly energy efficient. Also, along with the specific benefits of scalability they have efficient communication. The basic principle of a cluster-based protocol is to organize the sensor nodes into groups called clusters. In each cluster, a selected cluster head $(\mathrm{CH})$ is responsible for collecting the data from other members of the cluster, then aggregate the collected data, and further forward it to a base station. Using this principle, it is possible to reduce the amount of data transferred within the network, thus results in saving energy.

Clustering phenomenon, plays an important role in the organization of network which affect network performance. WSNs are several important limitations that should be consider for clustering schemes:

- Limited Energy: Limited energy in sensor node must be considered as clustering can reduce overall energy usage in a network.

- Network Lifetime: Energy limitation in nodes in a network results in a limited network life.

- Limited Abilities: Small physical size and a small amount of energy stored in the sensor nodes limits communications capabilities as well as processing capabilities of nodes.

- Application Dependency: When a clustering algorithm is designed, application consolidation is a good clustering algorithm should be considered as a variety of application needs to be able to adapt to.

\section{Motivation}

It was observed that wireless sensor networks have found their way into a wide variety of applications and systems with vastly varying requirements and characteristics. As a consequence, it was very difficult to discuss specific application requirements regarding hardware issues and software support. This is particularly problematic in a multidisciplinary research area such as wireless sensor networks, where close collaboration between users, application domain experts, hardware designers, and software developers is needed to implement efficient systems. So many issues and challenges is opened in this system. Thus it gives the motivation to address those challenges and improvement. The consequences of the design space of wireless sensor networks by considering various dimensions and proposed a recursive data dissemination protocol for wireless sensor networks. Energy efficiency is an important design consideration for these networks. Motivated by the fact that sensor network queries may often be geographical. Other issues such as dynamic and static cluster problems, Network Lifetime, Power Conservation. All these aspects gives greater motivation to do new research to overcome all these open issues.

\section{Reviews On Challenges Involved In WSN}

Chiara Buratti et.al.[1] aims at WSNs technologies, main applications and standards, also on WSNs design features, and an overview of the evolutions. In particular, environmental monitoring, such as those based on some strange applications are discussed and highlighted strategies designed; In a case study based on an actual implementation is also explained. Detect trends and possible evolutions. Emphasis IEEE 802.15.4 technology, which enables multiple applications of WSNs are given to. Some examples of 802.15.4 based network performance characteristics are shown and discussed as a function of size and data type of WSN nodes.

L. Malathi et.al.[2] Optimize parameters such as, clustering overhead, network life, energy hole, FND (first node dies) and LND (last node dies). WSN proposed a hybrid uneven clustering layering (HUCL) protocol. HUCL is a static and dynamic clustering approach which is combination of both and hence it is hybrid. 
In HUCL, the network layers are divided into groups of different sizes. Available energy of nodes is the basis for choosing the cluster head, and were also chosen based on the number of neighbors to the sink. Once the group is formed, the same structure is maintained for a few rounds. Data with a data compression algorithm in a multi-hop network layer-based communication is sent through to the sink. In comparison with existing protocols, HUCL balances energy and its groups achieves a good distribution in the network, extend the lifetime of the network and avoid the energy hole problem.

Ameer Ahmed Abbasi.et.al.[3] publishes a general classification of clustering schemes. Presenting different clustering algorithms for WSNs survey; Its objectives, features, complexity, etc. Also highlighted the convergence rate of such clustering, cluster stability, overlapping clusters, support for location awareness and node mobility as compared to algorithms based on metrics.

Giuseppe Anastasi a .et.al.[4] author presents the energy consumption of the components of a typical sensor node, and discussed the main directions for energy conservation in WSNs. However, energy conservation schemes, which are discussed in the depths in a systematic and comprehensive classification. Special attention promising solutions such as energy-efficient data acquisition techniques that have not yet received widespread attention in the literature has been devoted to. Finally research directions for energy conservation in WSNs are concluded.

Chengfa Li et.al.[5] proposes the energy-efficient unequal clustering (EEUC) mechanism for periodic data gathering in wireless sensor networks. The nodes split into groups of unequal size and the size of the group closer to the base station is smaller than those further away from the base station. Thus the nodes closer to the base station can preserve some energy for data forwarding in intercluster communication. Also an energy-aware multihop routing protocol for inter-cluster communication proposed. Simulation results show that uneven clustering mechanism well among all sensor nodes balances the energy consumption in the network also network lifetime achieves a clear improvement.

Jun Luo Jean-Pierre Hubaux et.al.[6] shows that the base station should have mobility so that sensors close to it change over time as they have to relay data due to which their batteries runs out quickly. If the mobility and multi-hop routing dynamics both are taken into account by the base station then data collection protocols can be optimized. It is conclude that the best perimeter of the network mobility strategy are (it is assumed that the sensors are deployed within a circle) consists in the periphery. So in this case jointly consider mobility and routing algorithms, routing round and a better strategy uses a combination of routes and minor roads show. For each of the statements provided a detailed analytical model, and simulation results confirm this. In terms of the lifetime of the network $500 \%$ improvement is achieved.

Dinu gopal et.al.[7] In this paper, a novel routing technology proposes routing schemes VGDRA and VCCSR for data collection. By using a hybrid scheme, it become possible to find a balance in energy consumption, average delay and lifetime. VGDRA reduce the cost of rebuilding of routes whereas VCCSR will reduce energy consumption and increase the lifetime of the network.

Yan Gu et.al.[8] propose a multi layered clustering algorithm for network to balance the energy between clusterheads. After that performance of this approach is evaluated with simulation. The result shows that this algorithm is energy efficient and also increases network lifetime.

Hairong Zhao et.al.[9] proposed a protocol for better establishment of Cluster and data transmission path. In the process of setting a cluster, a timer is introduced so that optimal sensor node should be elected as cluster head. During transmission of data, single-hop and multi-hop hybrid routing to communicate more effectively and evenly. An example simulation algorithm is provided to demonstrate the utility which greatly increase the network life and low energy consumption compared to LEACH algorithm.

Debashis De et.al.[10] is changing the transmission ranges of nodes temporarily for that "bubbling mechanism" is proposed here, the concept of "cluster border" in which clusters at the border are having more number of active nodes than internal clusters. We also increase the efficiency of data collection by using dual sink i.e static sink and multiple mobile sinks. Approximately $10.62 \%$ of the energy of loaded cluster head is saved by using this mechanism.

Rabia Noor Enam et.al.[11] In this paper, energy consumption has been calculated for the transmission of data from the sensor nodes to sink. It has been shown through extensive simulations that $20 \%$ of the total energy consumption of the network expires in the process of $\mathrm{CH}$ selection phase only. This energy consumption can be taken as an overhead power. An energy-efficient "round rotation" mechanism is proposed to overcome this overhead energy. By introducing RR technique with random $\mathrm{CH}$ selection phase, overhead energy of the network is reduced by $4 \%$ during data transmission. The result has been augmented by $20 \%$ of the network lifetime.

Zohare. Arabi et.al.[12] An algorithm for Hybrid energy efficient routing is proposed that uses the two algorithms, i.e EF- Tree (earliest-first tree) and SID (source- Initiated Dissemination) to disseminate the data and a fuzzy method is employed for selecting cluster heads, and to switch between Sid and EF- tree. In this way, the whole network is grouped into cluster and fuzzy method is used to choose cluster head according to 
fuzzy variables. However, analysis of changes in the fuzzy variables and fuzzy, then EF-tree or SID is selected for transmitting information. Simulation results indicate that HERF has improved energy efficiency.

Ananya Patra et.al.[13] In this paper, an energy-efficient hybrid clustering scheme (IEEHCS) is introduced which is a cluster formation algorithm and a selection process is also proposed here. In the proposed scheme, centralized algorithm is used for selection of energy efficient CHS which also depends upon remaining energy, node density and minimum separation distance between the nodes to reduce overheads. The main idea is that the $\mathrm{CH}$ role is repeated with the same settings again and again in each round and is shifted to an eligible member nodes instead of re-clustering of whole network. Thus, IEEHCS, reduces the frequency of updating the CHS in each round and avoids unnecessary re-clustering which saves a considerable amount of energy. Simulation results that IEEHCS effectively reduces energy consumption of energy and improves the lifetime of the network for the first node death by $45.39 \%$ and $11.36 \%$ compared to Leach-C and EEHCS respectively.

Rajesh Purkait et.al.[14] this paper address problem of hot spot and equal clustering problems and by considering the energy efficiency of WSN routing algorithms and unequal size clustering is proposed here. These algorithms are tested with different scenarios. Result shows that the proposed algorithm is better in terms of a no. of active of nodes in network, average energy consumption and network lifetime.

\section{Conclusion And Future Work}

Sensor networks that monitor environmental changes by adapting to the sensor are required to operate. The network must be self-learning. Reliability is to maintain sensor network functionalities without any interruption due to failure. Sensor node may fail due to lack of energy, physical damage, circulatory problems, inactivity, or may fail due to environmental interference. Network should be able to organize itself after detection of node failure. The model that we have in the future is energy efficient that will handle dynamic clustering and static clustering.

\section{References}

[1]. Chiara Buratti, Andrea Conti, Davide Dardari and Roberto Verdone, " An Overview on Wireless Sensor Networks Technology and Evolution" Sensors, Aug 2009, pp. 6869-6896.

[2]. L. Malathi , R.K. Gnanamurthy, Krishnan Chandrasekaran, " Energy efficient data collection through hybrid unequal clustering for wireless sensor networks" Elsevier, June 2015, pp. 1-18.

[3]. Ameer Ahmed Abbasi and Mohamed Younis, "A survey on clustering algorithms for wireless sensor networks" Elsevier, Computer Communications, June 2007, pp. 2826-2841.

[4]. Giuseppe Anastasi a, Marco Conti b, Mario Di Francesco and Andrea Passarella, "Energy conservation in wireless sensor networks: A survey" Elsevier, Ad Hoc Networks, July 2009, pp. 537-568.

[5]. Chengfa Li, Mao Ye, Guihai Chen and Jie Wu, " An Energy-Efficient Unequal Clustering Mechanism for Wireless Sensor Networks" supported by China Jiangsu Provincial NSF grant (BK2005208), China 973 project (2002CB312002) and TRAPOYT award of China Ministry of Education, and US NSF grants CCR 0329741, CNS 0422762, and CNS 0434533, pp. 1-8.

[6]. Jun Luo Jean-Pierre Hubaux, " Joint Mobility and Routing for Lifetime Elongation in Wireless Sensor Networks" IEEE INFOCOM, 2005, pp. 1-12.

[7]. Dinu Gopal and Suryakala C.D," Performance evaluation of hybrid energy efficient cluster based routing scheme for mobile sink based WSN" Control Instrumentation, Communication and Computational Technologies (ICCICCT), IEEE International Conference, 2015 ,pp. 558-564.

[8]. Yan Gu, Dahai Jing and Jie Guo," Energy Efficient Layered Clustering Approach for WSN "Control Engineering and Communication Technology (ICCECT), IEEE International Conference, 2012, pp. 628-631.

[9]. Hairong Zhao, Wuneng Zhou and Yan Gao," Energy Efficient and Cluster Based Routing Protocol for WSN" Computational Intelligence and Security (CIS), Eighth IEEE International Conference, Nov 2012, pp. 107-111.

[10]. Debashis De, Aditi Sen and Madhuparna Das Gupta," Cluster Based Energy Efficient Lifetime Improvement Mechanism for WSN with Multiple Mobile Sink and Single Static Sink" Computer and Communication Technology (ICCCT), Third IEEE International Conference, Nov2012, pp. 197-199.

[11]. Rabia Noor Enam, Syed Misbahuddin and Mumtazul Imam," Energy efficient round rotation method for a random cluster based WSN" Collaboration Technologies and Systems (CTS), IEEE International Conference, May 2012, pp. 157-163.

[12]. Zohre. Arabi,"HERF: A hybrid energy efficient routing using a fuzzy method in Wireless Sensor Networks" Intelligent and Advanced Systems (ICIAS), IEEE International Conference, Jun 2010, pp. 1-6.

[13]. Ananya Patra and Sonali Chouhan,"An approach to improved energy efficient hybrid clustering in wireless sensor networks" IEEE International Conference on Signal Processing and Communications (SPCOM), July 2014, pp.1-6.

[14]. Rajesh Purkait; Sachin Tripathi,"Energy efficient unequal clustering and routing algorithms for wireless sensor networks" IEEE Workshop on Computational Intelligence: Theories, Applications and Future Directions (WCI), Sep 2015. pp. 1-10.

[15]. Sukhchandan Randhawa, " Research Challenges in Wireless Sensor Network: A State of the Play" Conference Proceeding of National Conference on convergence of Science, Engineering \& Management in education and research, March 2014.

[16]. I.F. Akyildiz, W. Su, Y. Sankara subramaniam and E. Cayirci, "Wireless sensor networks: a survey" Elsevier, Computer Networks 38, Dec 2001, pp. 393-422.

[17]. Ian F. Akyildiz, Weilian Su, Yogesh Sankarasubramaniam, and Erdal Cayirci, "A Survey on Sensor Networks" IEEE Communications Magazine, August 2002, pp. 102-114.[23] Chalermek Intanagonwiwat, Deborah Estrin, Ramesh Govindan and John Heidemann, "Impact of Network Density on Data Aggregation in Wireless Sensor Networks " supported by the Defense Advanced Research Projects Agency under grant DABT63-99-1-0011, Nov 4, 2001, pp.1-16. 